\title{
Morphological aspects and deterministic reconstruction of dynamical fracture surfaces in brittle materials
}

\author{
D. Dalmas ${ }^{1, a}$, C. Guerra ${ }^{2,3}$, J. Scheibert ${ }^{2}$, and D. Bonamy ${ }^{2}$ \\ 1 Unité Mixte CNRS/Saint-Gobain "Surface du Verre et Interfaces" UMR 125, BP 135, F-93303, \\ Aubervilliers, Cedex, France. \\ 2 CEA, IRAMIS, SPCSI, Grp. Complex Systems \& Fracture, F91191 Gif sur Yvette, France. \\ 3 Universidad Autónoma de Nuevo Leon, San Nicolás de los Garza, México.
}

\section{Introduction}

Linear Elastic Fracture Mechanics (LEFM) provides a quantitative description of the crack motion that agrees well with observations in brittle materials as long as the crack growth is sufficiently slow. However, several issues remain unsolved: (i) - experimental observations reveal that at high velocity, the crack speed is systematically smaller than the one predicted by LEFM [1] and (ii) above a given velocity, a dynamically growing crack creates a structure of its own as evidenced from roughening of fracture surfaces at high speed [2]. The aim of this work is to present a statistical study and a deterministic reconstruction of the morphology of the fracture surfaces in Plexiglas obtained under dynamic conditions.

\section{Experimental set-up}

We have designed an experimental setup based on the wedge splitting geometry in which we control the dynamical crack propagation in opening mode in a model brittle materials: Plexiglas. Crack velocity is recorded in real time during fracture, and the morphology of fracture surfaces is observed post mortem by optical microscopy and profilometry. A large number of Plexiglas specimens were fractured. They were broken at various crack velocities in the range $100-500 \mathrm{~ms}^{-1}$, i.e. $0.1-0.5$ the Rayleigh wave speed. Post mortem analysis of the fracture surfaces were carried out in this crack velocity range.

\section{Results and analysis}

Post mortem examinations of the surfaces give us interesting clues about the events that have occurred during the fracture process. One can clearly see on Fig. 1, so called conic marks. At velocities lower than typically $\sim 150 \mathrm{~ms}^{-1}$ no conic mark is observed [3]. In this case we observe a "smooth" fracture surface. At higher velocities, the surface density of observed conics marks is found to increase with crack velocity [3]. This behaviour was already observed earlier by Ravi-Chandar and Yang [4] for instance. To go further in the analysis of this damage mechanisms, we have characterized and analyzed the statistics distribution of the nucleation site of these micro-cracks: spatial distribution, time delay, voronoï tesselation...

Each conic mark is believed [4] to result from the intersection of the main crack and a pennyshaped micro-crack nucleated ahead of it. In such model, the propagation dynamics is only of geometrical nature, provided that for each conic mark (i) the location of its focus and (ii) the distance

\footnotetext{
a e-mail: davy.dalmas@saint-gobain.com
} 

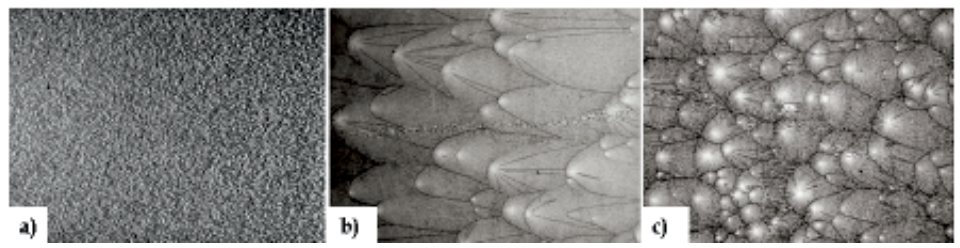

Fig. 1. Microscope images $\left(1.4 \times 1 \mathrm{~mm}^{2}\right)$ of the fracture surfaces, taken at : (a) $v=110 \pm 10 \mathrm{~ms}^{-1}$, (b) $v=$ $250 \pm 20 \mathrm{~ms}^{-1}$ (c) $v=450 \pm 50 \mathrm{~ms}^{-1}$. Crack propagation is from left to right.
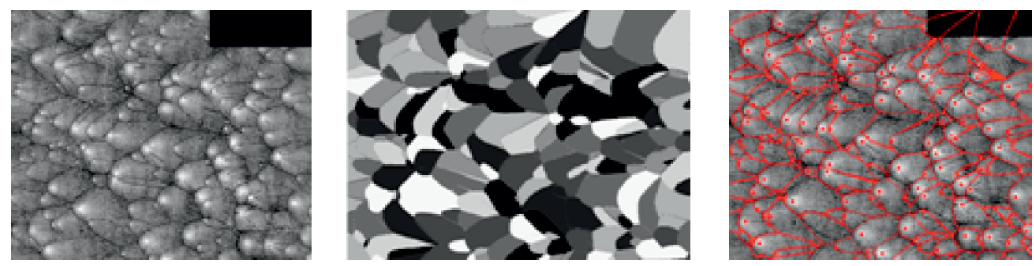

Fig. 2. Left side : Optical profilometer images taken from the fracture surfaces at velocities of $350 \mathrm{~ms}^{-1}$. Center : final image reconstruction of surface markings. Right : Superposition of the optical profiometer images vs. the reconstructions (red lines).

required for a neighbouring micro-crack front to trigger its opening are given. Based on this simple geometrical model, we have shown that it was possible to deterministically reconstruct the dynamics of propagation of the crack front and the development of associated damage from the fracture surface (Fig. 2).

\section{Conclusion}

The experimental work presented here in Plexiglas allows to relate the morphology of post-mortem fracture surfaces to the crack growth velocity. When this velocity is larger than $\sim 150 \mathrm{~ms}^{-1}$, one can observe conic markings on the surface. These markings are found to be the signature of the interaction between the main crack front and growing penny-shaped micro-cracks ahead. We have characterized the statistics of the nucleation site of these micro-cracks. The understanding of this damage mechanisms through a deterministic reconstruction shed light on the origin of the discrepancy between theory and experiments at large fracture velocities [5].

\section{References}

1. L.B. Freund, Dynamic Fracture Mechanics (Cambridge University Press, New York 1990).

2. B. Cotterell, J. R. Rice, International Journal of Fracture 16 (1980) 155 -169.

3. J. Scheibert, C. Guerra, F. Célarié, D. Dalmas, D. Bonamy, Phys. Rev. Lett. (to be published)

4. K. Ravi-Chandar, W.G. Knauss, International Journal of Fracture 26 (1984) 65- 80.

5. C. Guerra, J. Scheibert,D. Bonamy, D. Dalmas,(in preparation) 